\title{
Magnetic, Thermal, and Topographic Imaging with a Nanometer-Scale SQUID-On-Lever Scanning Probe
}

\author{
M. Wyss $\odot,{ }^{1,2}$ K. Bagani, ${ }^{1}$ D. Jetter $\odot,{ }^{1}$ E. Marchiori, ${ }^{1}$ A. Vervelaki, ${ }^{1}$ B. Gross, ${ }^{1}$ J. Ridderbos, ${ }^{1}$ \\ S. Gliga, ${ }^{3}$ C. Schönenberger $\odot,{ }^{1,2}$ and M. Poggio $\circledast^{1,2, *}$ \\ ${ }^{1}$ Department of Physics, University of Basel, 4056 Basel, Switzerland \\ ${ }^{2}$ Swiss Nanoscience Institute, University of Basel, 4056 Basel, Switzerland \\ ${ }^{3}$ Swiss Light Source, Paul Scherrer Institute, 5232 Villigen, Switzerland
}

(Received 16 September 2021; revised 2 December 2021; accepted 7 February 2022; published 1 March 2022)

\begin{abstract}
Scanning superconducting quantum interference device (SQUID) microscopy is a magnetic imaging technique combining high field sensitivity with nanometer-scale spatial resolution. Here, we demonstrate a scanning probe that combines the magnetic and thermal imaging provided by an on-tip SQUID with the tip-sample distance control and topographic contrast of a noncontact atomic force microscope (AFM). We pattern the nanometer-scale SQUID, including its weak-link Josephson junctions, via focused-ionbeam milling at the apex of a cantilever coated with $\mathrm{Nb}$, yielding a sensor with an effective diameter of $365 \mathrm{~nm}$, field sensitivity of $9.5 \mathrm{nT} / \sqrt{\mathrm{Hz}}$, and thermal sensitivity of $620 \mathrm{nK} / \sqrt{\mathrm{Hz}}$, operating in magnetic fields up to $1.0 \mathrm{~T}$. The resulting SQUID-on-lever probe is a robust AFM-like scanning probe that expands the reach of sensitive nanometer-scale magnetic and thermal imaging beyond what is currently possible.
\end{abstract}

DOI: 10.1103/PhysRevApplied.17.034002

\section{INTRODUCTION}

Nanometer-scale magnetic imaging techniques, based on optical, electron, x-ray, or scanning probe sensors, reveal magnetization patterns, spin configurations, and current distributions. They provide local information about length scales, inhomogeneity, and interactions that is inaccessible in bulk measurements of transport, magnetization, susceptibility, or heat capacity. Prominent among these techniques, for their combination of high sensitivity and high spatial resolution, are scanning probe microscopies such as magnetic force microscopy, scanning nitrogenvacancy center microscopy, and scanning superconducting quantum interference (SQUID) device microscopy [1]. Exploiting its intrinsic sensitivity to magnetic flux and its minimal interaction with the sample, researchers have used scanning SQUID microscopy to image a wide variety of nanometer-scale phenomena, including superconducting vortices [2-5], persistent currents in normal metal rings [6], magnetism and superconductivity at oxide interfaces [7,8], and magnetic reversal in nanomagnets $[9,10]$. Scanning SQUIDs have also been used to visualize edge currents in the quantum Hall [11] and quantum spin Hall [12] states, as well as twist-angle disorder [13] and orbital magnetism [14] in twisted bilayer graphene.

\footnotetext{
*martino.poggio@unibas.ch
}

In scanning SQUID microscopy, high spatial resolution is achieved by minimizing both sensor size and its distance from the sample. So far, two strategies have yielded the best results. The first uses standard processing techniques to produce a planar SQUID that includes a small pick-up loop near the corner of the chip, where it can be scanned close to a sample [15-17]. The second is to fabricate so-called SQUID-on-tip probes [18,19], in which a superconductor is evaporated or sputtered directly on the end of a pulled quartz tip, forming a SQUID. This method allows the fabrication of the smallest scanning sensors - down to $50 \mathrm{~nm}$ in diameter with a sensitivity of $5 \mathrm{nT} / \sqrt{\mathrm{Hz}}[20]$ and the closest approach to the sample. Because of this proximity to the sample, the Josephson junctions (JJs) in the SQUID loop can also be used for thermal microscopy with a sensitivity better than $0.5 \mu \mathrm{K} / \sqrt{\mathrm{Hz}}$ [21]. Such sensitive thermal microscopy was recently used to visualize dissipation in the quantum Hall state in graphene [22]. However, the process for fabricating a SQUID-on-tip probe limits its complexity, e.g., it precludes the integration of field coils for susceptibility measurements or flux feedback, as is done in the planar scheme [23]. Also, because of the intrinsic stiffness of both the planar and SQUID-on-tip probes, neither allows simultaneous imaging of the sample topography. Although fragile SQUID-on-tip sensors are typically coupled to tuning fork resonators to avoid contact with the sample, they are only sensitive to topography within tens of nanometers 
(a)

(i)

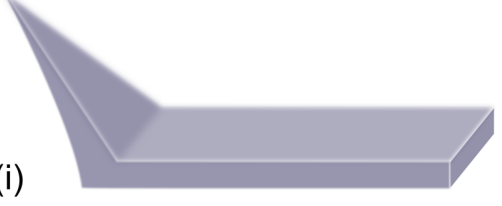

(ii)

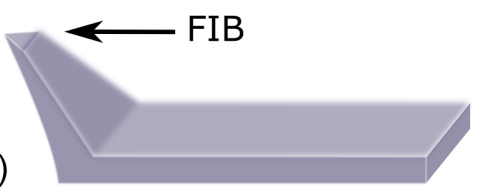

(iii)

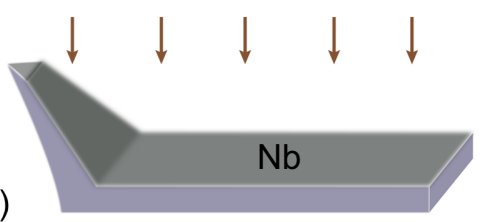

(iv)

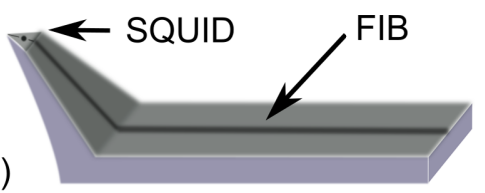

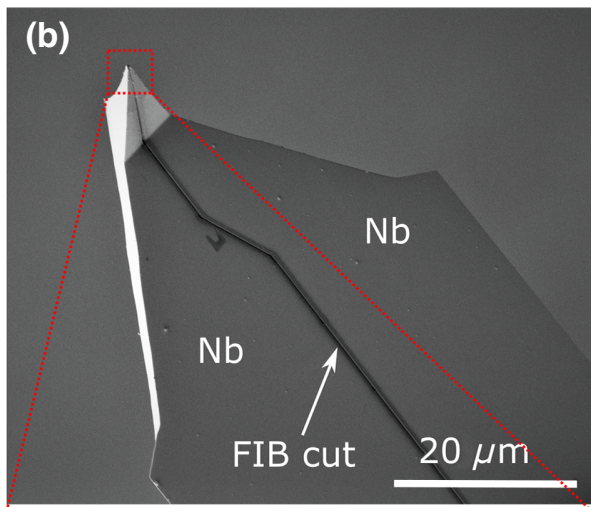

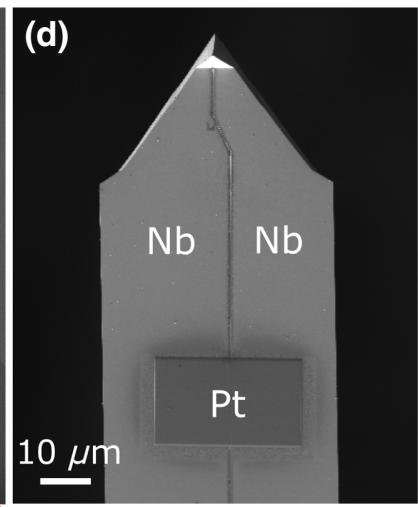

(e)

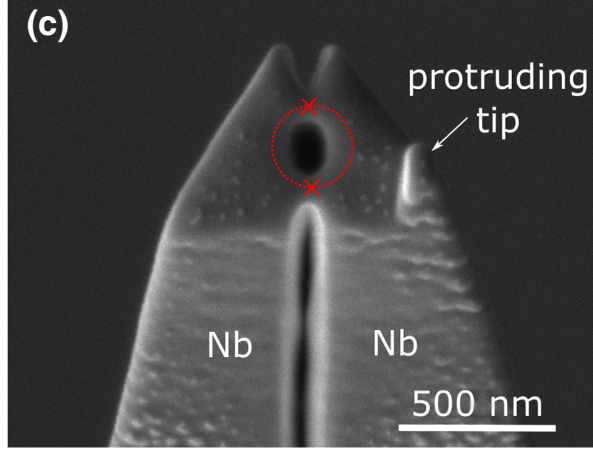

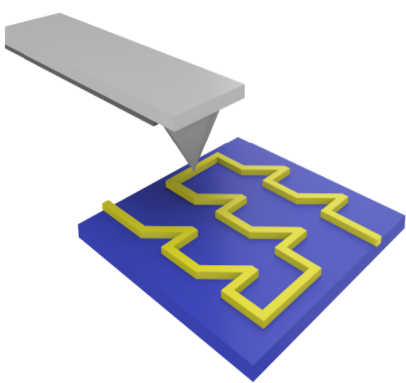

FIG. 1. SQUID-on-lever fabrication. (a) Schematic of patterning steps. (i) The tip of an AFM cantilever (ii) is milled away, leaving a triangular plateau. (iii) A Nb film is deposited on the milled side of the cantilever and (iv) is milled via FIB to define a SQUID. (b) SEM image of the cantilever showing the $\mathrm{Nb}$ film and the FIB-milled trench separating two leads to the on-tip device. (c) Enlarged SEM image showing the FIB-milled trenches and hole defining the SQUID device. (d) Pt shunt resistance connecting two Nb leads. (e) Schematic of the scanning probe and sample.

from the surface, making imaging or stabilization of the tip-sample distance challenging.

Here, we use focused-ion-beam (FIB) milling to fabricate a nanometer-scale SQUID at the apex of a cantilever force sensor, combining the capabilities of a SQUID-on-tip probe with those of noncontact atomic force microscopy (AFM). All components of the SQUID, including superconducting leads, loop, and weak-link JJs, are patterned via direct FIB milling of a sputtered $\mathrm{Nb}$ film, overcoming the challenge of patterning directly on a high-aspect-ratio tip. FIB patterning offers flexibility in the possible designs of the SQUID, allowing for the inclusion of modulation lines and field coils, as in planar pick-up loop devices. The result is a SQUID-on-lever scanning probe that provides sensitive magnetic, thermal, and topographic contrast. In addition, the noncontact AFM cantilever supplies excellent control of the tip-sample spacing, even for tip-sample spacings exceeding $1 \mu \mathrm{m}$.

\section{SQUID-ON-LEVER FABRICATION AND SETUP}

The fabrication of a SQUID at the apex of a commercially available $\mathrm{Si}$ cantilever designed for noncontact AFM is presented schematically in Fig. 1(a). We use a $\mathrm{Ga}^{+}$FIB to mill away the tip of the cantilever, leaving a triangular plateau. As part of this process, a small protrusion is left on the side of the plateau, as seen in the scanning electron microscope (SEM) image of Fig. 1(c). Its role is both as a sharp tip to optimize spatial resolution in AFM mode and to prevent the SQUID from touching the sample during scanning. Next, we deposit a 50-nm film of $\mathrm{Nb}$ on the milled side of the cantilever. We then pattern the SQUID on the coated cantilever via direct milling of the $\mathrm{Nb}$ film with a $\mathrm{Ga}^{+}$FIB. This process entails first cutting a long trench in the film through the middle of the cantilever. This FIB-cut trench, shown in Fig. 1(b), defines two electrical contacts running from the base of the cantilever to its apex. We then mill a hole in the center of the triangular plateau followed by a second, shorter trench. The hole separates the long and short trenches and together they define two Dayem bridge JJs and hence a Nb SQUID at the tip of the cantilever. Figure 1(c) shows such a SQUID with a diameter of $300 \mathrm{~nm}$ and a protruding tip of height 175 $\mathrm{nm}$. Finally, a thin film of Pt is patterned via FIB-induced deposition to serve as a shunt resistor between the two $\mathrm{Nb}$ leads, as shown in Fig. 1(d).

The resulting SQUID-on-lever probe is mounted in a custom-built scanning probe microscope operating under vacuum at $4.2 \mathrm{~K}$. Piezoelectric positioners move the sample under the cantilever with nanometer-scale 
(a)

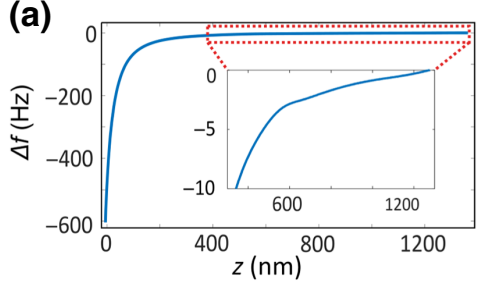

(b)

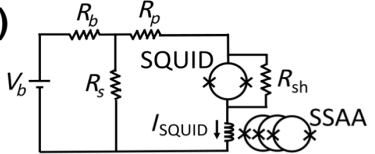

(c)

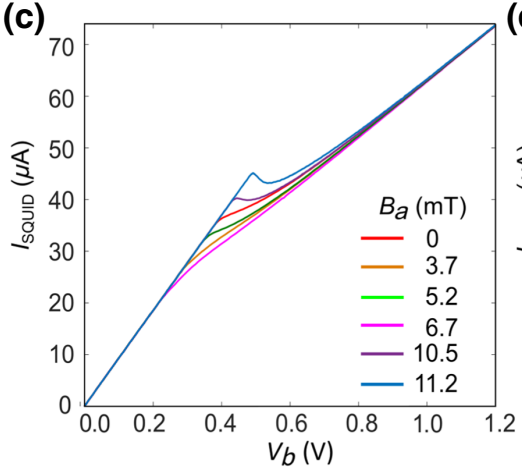

(f)
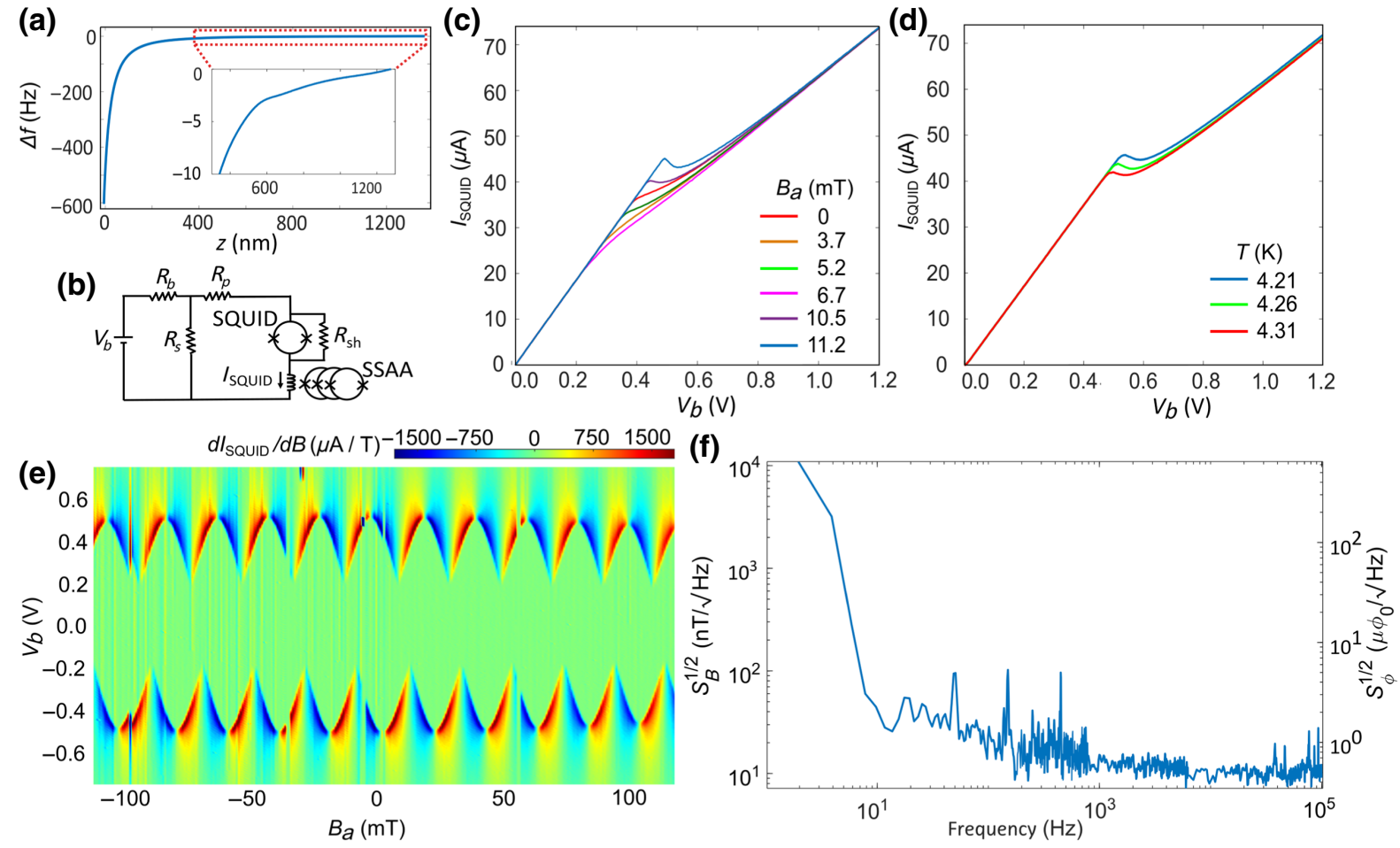

FIG. 2. SQUID-on-lever characterization. (a) $\Delta f(z)$ curve showing an approach of the cantilever to the sample surface. (b) Schematic of the measurement circuit including inductive coupling to a SSAA, where $R_{b}=6.1 \mathrm{k} \Omega, R_{s}=3 \Omega, R_{p}=2 \Omega, R_{\mathrm{sh}}=4 \Omega$. (c) $I_{\mathrm{SQUID}}\left(V_{b}\right)$ for the $\mathrm{Nb}$ SQUID-on-lever probe measured at $4.2 \mathrm{~K}$ and at different $B_{a}$ applied along the axis of the SQUID. (d) $I_{\text {SQUID }}\left(V_{b}\right)$ measured at different $T$ and $B_{a}=0$. (e) Magnetic response $d I_{\text {SQUID }} / d B$ of the SQUID as a function of both $V_{b}$ and $B_{a}$, showing a quantum interference pattern. (f) Power spectral density of the device at $4.2 \mathrm{~K}$, in terms of both magnetic field (left) and flux quanta (right). White noise from the device reaches $0.48 \mu \Phi_{0} / \sqrt{\mathrm{Hz}}$ or $9.5 \mathrm{nT} / \sqrt{\mathrm{Hz}}$.

precision and a specialized cantilever holder allows for both electrical contact to the SQUID-on-lever probe as well as mechanical excitation. The cantilever's flexural motion is driven at its fundamental resonance frequency $f_{0}$ of around $300 \mathrm{kHz}$ by a piezoelectric disk and is detected using a fiber-optic interferometer. As the cantilever approaches the substrate, $f_{0}$ shifts, due to tip-sample interaction. This shift $\Delta f$ is plotted as a function of tipsample distance $z$ in Fig. 2(a). The inset of Fig. 2(a) shows a significant response in $\Delta f$ even for $z>1 \mu \mathrm{m}$. As in standard noncontact AFM, this interaction enables a controlled approach, as well as the ability to safely scan within a few tens of nanometers of the sample surface by stabilizing at a constant $\Delta f$.

\section{MAGNETIC AND THERMAL SENSITIVITY}

We characterize the magnetic and thermal sensitivity of the SQUID-on-lever probe at $4.2 \mathrm{~K}$ using the circuit in Fig. 2(b). We operate the SQUID in voltage-bias mode $[20,24]$ with a large bias resistor $R_{b}$ and a small shunt resistor $R_{s}$. The circuit includes a parasitic resistance $R_{p}$ in series with the SQUID due to the wires and contacts. The Pt shunt resistance $R_{\mathrm{sh}}$ is connected in parallel with the SQUID to reduce hysteresis in the current through the
SQUID $I_{\text {SQUID }}$, by damping its high-frequency dynamics. We measure $I_{\text {SQUID }}$ using a commercial cold SQUID series array amplifier (SSAA) (Magnicon) working in feedback mode. Figures 2(c) and 2(d) show $I_{\text {SQUID }}$ as a function of bias voltage $V_{b}$ measured at different applied magnetic fields $B_{a}$ and temperatures $T$, respectively. The initial linear dependence of $I_{\text {SQUID }}\left(V_{b}\right)$ is due to the resistances in the measurement circuit and is followed by a reduction in slope at the critical current $I_{c}$, when the SQUID enters the normal state. The measurements show a maximum $I_{c}=45 \mu \mathrm{A}$ with a modulation of up to $25 \mu \mathrm{A}$ produced by $B_{a}$ applied along the axis of the SQUID loop $\hat{z}$ at $T=4.2$ $\mathrm{K}$ and a reduction with increasing $T$. The critical temperature $T_{c}$ of the device is measured to be $5.8 \mathrm{~K}$, in principle allowing for its operation at lower temperatures [25]. We measure a higher $T_{c}=8.8 \mathrm{~K}$ in regions of the device that are not exposed to the FIB, i.e., where the $\mathrm{Nb}$ film is not contaminated by implanted $\mathrm{Ga}^{+}$ions.

Figure 2(e) shows the magnetic response function $d I_{\text {SQUID }} / d B$, where $B$ is the total magnetic field, as a function of $V_{b}$ and $B_{a}$. Pronounced quantum interference persists up to $B_{a}=1.0 \mathrm{~T}$ [25] with a period of $20 \mathrm{mT}$. This period corresponds to an effective SQUID diameter $d_{e}=365 \mathrm{~nm}$, which is slightly larger than the physical diameter measured in the SEM image of Fig. 1(c). This 
mismatch is due to both magnetic field lensing by the superconductor on the triangular plateau and the suppression of superconductivity in $\mathrm{Nb}$ caused by ion implantation near the milled regions. The interference pattern shows an asymmetry between positive and negative $V_{b}$, due to the asymmetry of the two JJs. By appropriate choice of $V_{b}$, this asymmetry increases the range of applied fields in which the SQUID is sensitive, including at $B_{a}=0$.

Figure 2(f) shows the spectral density of the SQUID's noise in terms of both magnetic flux and magnetic field measured at $B_{a}=0$ and $V_{b}=0.5 \mathrm{~V}$. The spectrum is dominated by $1 / f$-like noise at low frequencies and white noise less than $1 \mu \Phi_{0} / \sqrt{\mathrm{Hz}}$ above a few hundred hertz. At $10 \mathrm{kHz}$ and in the sensitive regions of the magnetic response function, the flux sensitivity reaches $S_{\Phi}^{1 / 2}=$ $0.48 \mu \Phi_{0} / \sqrt{\mathrm{Hz}}$, which corresponds to a magnetic field sensitivity $S_{B}^{1 / 2}=9.5 \mathrm{nT} / \sqrt{\mathrm{Hz}}$. The flux sensitivity is comparable to that of micrometer-scale planar scanning SQUIDs shown by Huber et al. [24] and better than that of previously reported submicrometer SQUID-on-tip probes made from Al [18], $\mathrm{Nb}$ [19], and $\mathrm{Mo}_{66} \mathrm{Re}_{34}$ [26]. The device does not, however, attain the flux sensitivity of Pb SQUID-on-tips [20]. Nevertheless, given that the measured sensitivity exceeds estimates for both the thermal $\left(120 \mathrm{n} \Phi_{0} / \sqrt{\mathrm{Hz}}\right)$ and quantum $\left(27 \mathrm{n} \Phi_{0} / \sqrt{\mathrm{Hz}}\right)$ noise [25], it is likely limited by interference and electrical noise and thus could be improved.

We determine the thermal sensitivity of the device by analyzing $I_{\mathrm{SQUID}}\left(V_{b}\right)$ measured at different $T$, as in Fig. 2(d). For a fixed $V_{b}=0.55 \mathrm{~V}$, the thermal response at $B_{a}=0$ and $T=4.2 \mathrm{~K}$ is $d I_{\mathrm{SQUID}} / d T=-24.2 \mu \mathrm{A} / \mathrm{K}$. Combining this response with the measured white current noise of the device yields a thermal sensitivity better than $620 \mathrm{nK} / \sqrt{\mathrm{Hz}}$, which is comparable to that of state-of-the-art $\mathrm{Pb}$ SQUID-on-tip sensors.

\section{IMAGING A CURRENT-CARRYING WIRE}

In order to demonstrate the capabilities of the SQUIDon-lever as a scanning probe, we first image a currentcarrying $\mathrm{Au}$ wire patterned on a Si substrate. We image the topography of the wire with our SQUID-on-lever probe by implementing a standard noncontact AFM technique: scanning in the $x y$ plane, while feeding back a correction $\Delta z$ to the piezoelectric scanner that controls the tip-sample spacing $z$, in order to maintain a constant frequency shift $\Delta f$ (constant-frequency mode). Figure 3(b) shows an AFM image of the Au wire scanned at a constant $\Delta f=-5 \mathrm{~Hz}$, which corresponds to $z=140 \mathrm{~nm}$. The low spatial resolution of the image is set by the small $\Delta f$ set-point resulting in a large tip-sample spacing [a higher-resolution topographic image is presented in Fig. 4(a)].
By measuring the SQUID response while scanning over the same area at a fixed height corresponding to $z=345$ $\mathrm{nm}$ above the substrate, we image the Biot-Savart field and the thermal dissipation produced by alternating current (ac) flowing through the wire. Note that the SQUID-tosample spacing $z_{\text {SQUID }}=z+175 \mathrm{~nm}$, due to the height of the protruding tip. Figure 3(c) shows the $z$ component of the Biot-Savart field $B_{z}^{\text {ac }}$ produced by $I_{\mathrm{ac}}=100 \mu \mathrm{A}$ of current at $f_{\text {ac }}=4.17 \mathrm{kHz}$. Sensitivity to magnetic field is achieved by choosing a combination of $B_{a}$ and $V_{b}$, at which the SQUID-on-lever probe has a strong magnetic response [plotted in Fig. 2(e)], and measuring its first harmonic response at $f_{\mathrm{ac}}$.

By introducing a few millibars of ${ }^{4} \mathrm{He}$ exchange gas into the vacuum chamber, we also make our SQUID-onlever probe sensitive to the local temperature modulation $T_{\mathrm{ac}}$ produced by Joule heating as ac flows through the $\mathrm{Au}$ wire. The ability to sensitively map $T_{\mathrm{ac}}$ is made possible by the thermal coupling of the sensor to the sample. In particular, this exchange-gas-mediated coupling must be stronger than the coupling of the sensor to its support. To be a noninvasive probe, this coupling must also be weaker than the coupling of the sample with its substrate. Both of these conditions are met in our system, where the sensor is weakly coupled to its support, because of the small geometrical cross section of the cantilever and the absence of thermal conductivity along the superconducting film. Because $T_{\mathrm{ac}} \ll T$, where the bath temperature $T=4.2 \mathrm{~K}$, $T_{\mathrm{ac}}$ is in the small-signal limit and is proportional to the power dissipated, i.e., the square of the flowing current [21]. Therefore, to image $T_{\mathrm{ac}}$, we map the second harmonic response of the SQUID to the current at $2 f_{\text {ac }}$ [21], as shown in Fig. 3(d) with $I_{\mathrm{ac}}=100 \mu \mathrm{A}$.

Figure 3(e) shows another imaging mode available to the SQUID-on-lever probe: we actuate the cantilever's fundamental mode at $f_{0}=282 \mathrm{kHz}$ with an amplitude of $15 \mathrm{~nm}$ and measure the $z$ component of the magnetic field at this frequency $B_{z}^{\text {lever }}$. The resulting image, measured at a constant height corresponding to $z=345 \mathrm{~nm}$ over the substrate, is proportional to the spatial derivative of the magnetic field along the cantilever oscillation direction $d B_{z} / d z$ produced by $I_{\mathrm{dc}}=200 \mu \mathrm{A}$ of direct current (dc) flowing through the wire. The use of a lock-in amplifier to demodulate and spectrally filter the resulting signal substantially reduces $1 / f$ noise, which dominates dc measurements of $B_{z}$. Note that the background noise in Fig. 3(e) is about an order of magnitude larger than the $8 \mathrm{nT}$ expected from the white noise limit and the measurement bandwidth $(9.5 \mathrm{nT} / \sqrt{\mathrm{Hz}}$ and $830 \mathrm{mHz}$, respectively), because this measurement is not optimized to shield interference and minimize electronic noise, as is the one shown in Fig. 2(f). Imaging such spatial magnetic field derivatives also increases the maximum sensitivity towards smaller features sizes, compared with imaging magnetic fields [1]. In fact, detection of magnetic spatial derivatives is 

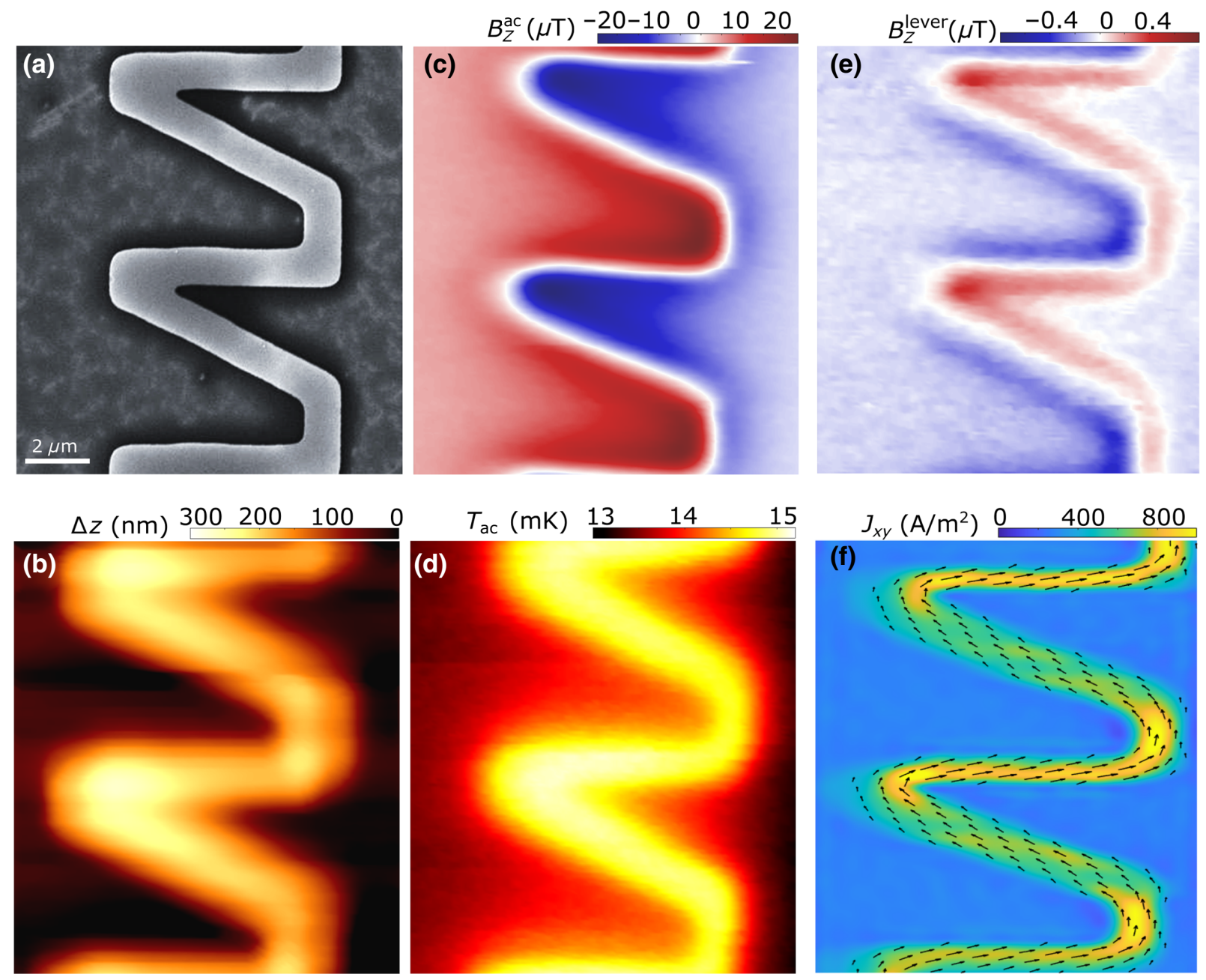

FIG. 3. Imaging a current-carrying wire. (a) SEM image of a 750-nm-wide and 120-nm-thick sawtooth-shaped Au wire patterned on a Si substrate. (b) Noncontact AFM image taken using the SQUID-on-lever probe in constant-frequency mode showing the sample topography $\Delta z$ measured at a tip-sample spacing $z=140 \mathrm{~nm}$. Scanning probe images made by the SQUID-on-lever probe at constant height from the substrate corresponding to $z=345 \mathrm{~nm}$ of (c) $B_{z}^{\mathrm{ac}}$ and (d) $T_{\mathrm{ac}}$ produced by $I_{\mathrm{ac}}=100 \mu \mathrm{A}$ flowing through the wire. (e) Image of $B_{z}^{\text {lever }}$ at the cantilever oscillation frequency produced by $I_{\mathrm{dc}}=200 \mu \mathrm{A}$ of dc. This signal is proportional to $d B_{z} / d z$. (f) $\mathrm{A}$ map of $\mathbf{J}_{x y}$ flowing through the wire calculated from the measurement of $B_{z}^{\text {ac }}$ in (c). Color scale indicates the magnitude and arrows the direction of $\mathbf{J}_{x y}$.

the standard mode for magnetic force microscopy and has also been implemented using a tuning fork resonator in scanning SQUID-on-tip microscopy [11] to increase sensitivity to small dc features in $B_{z}$ or local $T$.

The image of $B_{z}^{\text {ac }}$, shown in Fig. 3(c), can be reconstructed into a map of current density $\mathbf{J}_{x y}$ by inverting the Biot-Savart law [27,28]. Although three-dimensional current densities do not produce unique magnetic field patterns and can therefore not be determined by magnetic field imaging alone, in-plane current densities or current densities that are uniform throughout the thickness, do. By assuming a uniform $\mathbf{J}_{x y}$ throughout the thickness of the $\mathrm{Au}$ wire, we reconstruct the current density shown in Fig. 3(f).

\section{IMAGING AN ARRAY OF NANOMAGNETS}

In order to demonstrate the probe's applicability to nanomagnetic samples, we image an artificial spin ice $[29,30]$, shown in Fig. 4(a), that consists of a lattice of lithographically patterned nanometer-scale magnets, arranged in a square geometry, which exhibits magnetic frustration. Such systems are characterized by collective excitations, including emergent magnetic monopoles [29, 31-34] and magnonic modes [35-37]. Figure 4(b) shows the sample's topography imaged using the SQUID-onlever probe in constant-frequency mode with a set-point of $\Delta f=-120 \mathrm{~Hz}$, corresponding to $z=65 \mathrm{~nm}$. The resulting AFM image shows the nanomagnets with a resolution of the order of $50 \mathrm{~nm}$. The brightest and highest-resolution 

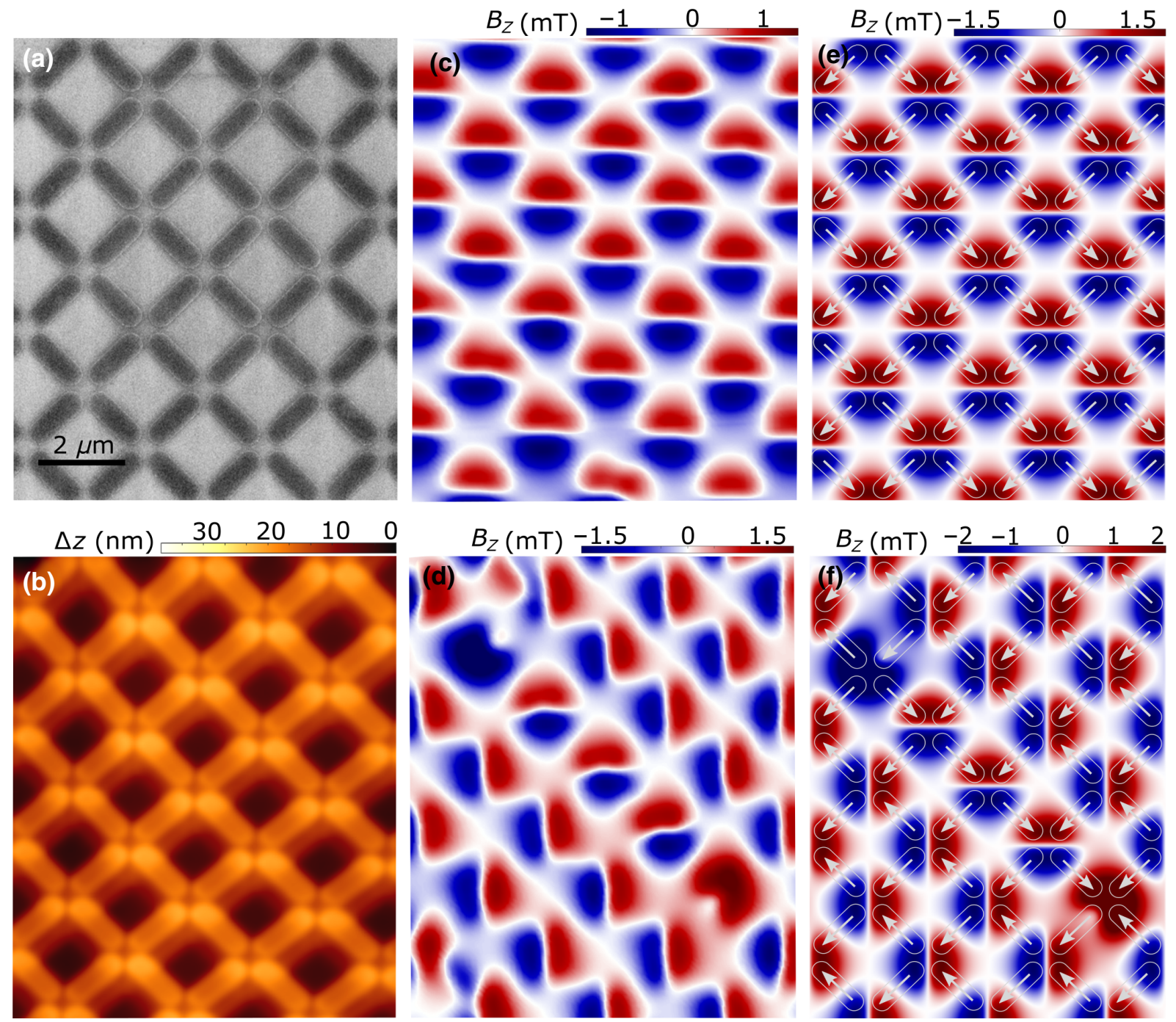

FIG. 4. Imaging artificial spin ice. (a) SEM image of a nanomagnet array consisting of a square lattice of 10-nm-thick Permalloy islands with a lateral size of $550 \times 1550 \mathrm{~nm}$ patterned on a Si substrate. (b) Noncontact AFM image taken using the SQUID-on-lever probe in constant-frequency mode showing the sample topography $\Delta z$ at a tip-sample spacing $z=65 \mathrm{~nm}$. (c) $B_{z}$ measured by the scanning SQUID-on-lever probe at constant height corresponding to $z=275 \mathrm{~nm}$ from the substrate. The spin ice is in remanence after saturation along $\hat{y}$ and (d) after the execution of a minor hysteresis loop, showing the signatures of separated magnetic charges and their corresponding numerical simulations (e),(f). The position of the nanomagnets is shown schematically and arrows show the orientation of their magnetic moment.

features in the image are due to the protruding tip patterned on the SQUID-on-lever probe, shown in Fig. 1(c). Fainter and lower-resolution "shadows" correspond to the sample's interaction with the larger and more distant plateau section of the tip, on which the SQUID is patterned.

Figure 4(c) shows a map taken at a constant height corresponding to $z=275 \mathrm{~nm}$ above the substrate $\left(z_{\text {SQUID }}=450\right.$ $\mathrm{nm}$ ) over the same region of the sample. We measure the SQUID response $I_{\text {SQUID }}$ to the magnetic stray field $B_{z}$ produced by the spin ice at remanence following saturation by an applied magnetic field along $\hat{y}, B_{a y}=100$ $\mathrm{mT}$. This corresponds to a two-in-two-out, type-II, state of the square ice. The measurement is in good agreement with the expected $B_{z}(x, y)$ obtained from corresponding micromagnetic simulations, shown in Fig. 4(e). The position and magnetization of the simulated islands is overlaid onto this map. Following a minor hysteresis loop (applying a magnetic field $B_{a x}=9.3 \mathrm{mT}$ and then reducing to zero), we record another image of the same region, shown in Fig. 4(d). Whereas magnetic charges are compensated at each four-nanomagnet vertex in the type-II configuration [Figs. 4(c) and 4(e)], after the minor loop, two vertices are left with uncompensated charges [Fig. 4(d)]. A string of vertices with compensated charges, whose distribution is rotated with respect to the rest of the array, 
connect these poles. Figure 4(f) shows the results of corresponding micromagnetic simulations matching the measurements. The orientation of each nanomagnet's magnetic moment reveals that the poles correspond to "monopoles" or type-III vertices. The asymmetric distribution of the stray fields around these points is due to the three-in-oneout or one-in-three-out configuration of the moments at the vertices.

\section{CONCLUSION}

The SQUID-on-lever probe's ultimate spatial resolution is limited by its effective size, which is different for each type of contrast. The roughly $50-\mathrm{nm}$ spatial topographic resolution demonstrated in Fig. 4(b) is limited by the sharpness of the FIB-milled protruding tip. The magnetic image shown in Fig. 4(c) can be reproduced by convolving the calculated magnetic field map of Fig. 4(e) with a Gaussian point-spread function of full width at half maximum $\mathrm{FWHM} \simeq 420 \mathrm{~nm}[25]$. This magnetic spatial resolution is close to, but larger than, $d_{e}=365 \mathrm{~nm}$ of the SQUID and smaller than the $900-\mathrm{nm}$ width of the triangular superconducting plateau. Upon close approach, the thermal imaging resolution should be limited by the distance between the JJs of around $300 \mathrm{~nm}$ [21]. The resolution exceeding $1 \mu \mathrm{m}$ apparent in Fig. 3(b), which is measured well beyond this regime at $z_{\text {SQUID }}=520 \mathrm{~nm}$, is limited by the heat transfer across the exchange gas, as discussed in Ref. [21].

Previous attempts at realizing on-tip SQUIDs have involved either micrometer-sized sensors and/or coupling to stiff tuning fork resonators, which preclude topographic contrast or tip-sample stabilization for spacings larger than tens of nanometers [17,19,38,39]. For this reason, the integration of a SQUID onto a conventional AFM cantilever greatly expands the range of samples that can be studied, including samples with significant topography. The main obstacle to realizing a nanometer-scale SQUID-on-lever probe has been the difficulty of patterning a tiny superconducting circuit on a high-aspect-ratio tip. Our work shows that FIB patterning - with its ability to mill superconducting materials on the nanometer scale and on nonplanar surfaces - provides a solution to this technical challenge. In addition, it may be possible to pattern even smaller SQUID-on-lever devices via FIB- or focused-electron-beam-induced deposition of superconducting nanostructures, e.g., from WC [40], or via the structural modification of superconductors via a $\mathrm{He}^{+}$FIB, e.g., as demonstrated in yttrium barium copper oxide [41]. Moreover, more complex SQUID devices than those demonstrated here, including modulation lines or coils for susceptibility measurements, could also be patterned using FIB milling. Such increased device complexity could significantly expand the capabilities of scanning probe microscopy, which-although spectacularly successful - is typically carried out with simple probes, such as sharp conducting, insulating, or magnetic tips.

\section{ACKNOWLEDGMENTS}

We thank Roy Haller for contributions to the sputtering of high-quality $\mathrm{Nb}$ and José María De Teresa for important suggestions. We also thank Sascha Martin and his team in the machine shop of the Physics Department at the University of Basel for help in building the measurement system. Some calculations were performed at sciCORE [42] scientific computing center at the University of Basel. We acknowledge the European Commission under H2020 FET Open grant "FIBsuperProbes" (Grant No. 892427), H2020 ERC Advanced grant TopSupra, the SNF under Grant No. 200020-178863 and under Spark project no. 190736, the Swiss Nanoscience Institute, the Kanton Aargau, and the NCCR Quantum Science and Technology (QSIT).

\section{APPENDIX A: EXPERIMENTAL METHODS}

\section{SQUID-on-lever fabrication}

The noncontact AFM cantilever (Nanosensors ATECNC) used here is made from doped single-crystal Si and has a fundamental mechanical resonance frequency $f_{0}=$ $335 \mathrm{kHz}$, spring constant $k=45 \mathrm{~N} / \mathrm{m}$, length $l=160 \mu \mathrm{m}$, widths $w=45 \mu \mathrm{m}$, and thickness $t=4.6 \mu \mathrm{m}$. We deposit $5 \mathrm{~nm}$ of $\mathrm{Ti}, 50 \mathrm{~nm}$ of $\mathrm{Nb}$, and $2 \mathrm{~nm}$ of $\mathrm{Pt}$ on the front side of the cantilever via magnetron sputtering. $\mathrm{Ti}$ and $\mathrm{Pt}$ are used as sticking and protection layers, respectively. Prior to sputtering, the chamber is pumped to a pressure of $5 \times 10^{-10}$ mbar. A dc power of $200 \mathrm{~W}$ is used during sputtering with a flow of $40 \mathrm{sccm}$ of Ar gas and a pressure of 4 mbar. After sputtering, we evaporate a 10 -nm-thick layer of $\mathrm{Au}$ to reduce ion implantation in subsequent FIBmilling steps. FIB milling is done in a dual-beam SEM and FIB (FEI Helios Nano Lab 650) in a pressure of $1 \times 10^{-5}$ mbar. Milling of the cantilever tip into a triangular plateau is done with a beam current of $1.1 \mathrm{pA}$. Milling of the trenches is carried out in five steps with different currents optimized to protect the $\mathrm{Nb}$ from ion implantation. For the long trench, far from the device area, a beam voltage of 30 $\mathrm{kV}$ and beam current of $0.4 \mathrm{nA}$ are used. Closer to the final device, the beam current is reduced in steps down to $1.1 \mathrm{pA}$ at the plateau. The rectangular Pt shunt resistance, shown in Fig. 1(d) of the main text, measures $30 \times 20 \mu \mathrm{m}^{2}$, is 20 -nm thick, and is patterned via FIB-induced deposition using a voltage of $30 \mathrm{kV}$ and beam current of $80 \mathrm{pA}$.

\section{Sample fabrication}

The current-carrying Au wire is patterned on a Si substrate using electron-beam lithography and deposited via electron-beam evaporation. The artificial spin ice, consisting of an array of permalloy $\left(\mathrm{Ni}_{83} \mathrm{Fe}_{17}\right)$ nanomagnets in 
a square ice geometry, is fabricated on a $\mathrm{Si}$ (100) substrate via electron-beam lithography, thermal evaporation at room temperature under a pressure of $10^{-6} \mathrm{mbar}$, and lift-off. The nanomagnets are $1.55-\mu \mathrm{m}$ long, $560-\mathrm{nm}$ wide, and have a lattice constant of $1.5 \mu \mathrm{m}$. A 2-nm-thick Al capping layer is evaporated on top of the permalloy to prevent oxidation. The thickness of the nanomagnets is measured by AFM to be $10 \mathrm{~nm}$ on average across the entire array, without taking the capping layer into account. The thickness is chosen to ensure that the nanomagnets are in a single-domain state [10].

\section{Scanning probe microscopy}

The scanning probe microscope operates under high vacuum in a ${ }^{4} \mathrm{He}$ cryostat and employs piezoelectric walkers and scanners (Attocube ANPx311/LT/HV, ANSxy 1001r/LT/HV) to move the sample. The fiber-optic interferometer consists of a 1550-nm diode laser, a 95:5 fiber-optic coupler, and a custom-built objective focusing light onto the cantilever to an $8-\mu \mathrm{m}$ spot. This low-finesse Fabry-Perot interferometer acts as a sensitive sensor of the cantilever's flexural motion, where the interference intensity is measured by a fast photoreceiver with an effective 3-dB bandwidth of $800 \mathrm{kHz}$. The cavity is stabilized against drift using a proportional-integral-derivative controller (Zurich Instruments MFLI) controlling the laser temperature. The incident power of around $1 \mu \mathrm{W}$ does not significantly heat the cantilever, as confirmed by measurements of laser power dependence and mechanical thermal motion. During approach and scanning, a phaselocked loop (Zurich Instruments MFLI) is used to excite and determine the cantilever's mechanical resonance frequency $f_{0}$.

\section{Micromagnetic simulations}

Micromagnetic simulations are performed with the Mumax $^{3}$ software package [43], which employs the Landau-Lifshitz-Gilbert micromagnetic formalism using finite-difference discretization. The size and shape of the simulated nanomagnets correspond to those of the measured spin ice sample. We use typical material parameters for Permalloy: saturation magnetization $\mu_{0} M_{S}=1 \mathrm{~T}$, exchange constant $A_{\mathrm{ex}}=1.3 \times 10^{-11} \mathrm{~J} / \mathrm{m}$, and no magnetocrystalline anisotropy, $K=0$. A temperature $T=0$ is assumed. The structure is discretized into cells of size $5.5 \mathrm{~nm} \times 5.5 \mathrm{~nm} \times 10 \mathrm{~nm}$.

\section{APPENDIX B: SENSOR CHARACTERIZATION}

\section{1. $T_{c}$ of the SQUID-on-lever probe}

The SQUID-on-lever probe is mounted on a custommade holder for scanning and its two superconducting leads are connected to sockets via wire bonds. Electrical contact is made to the socket for characterization of the

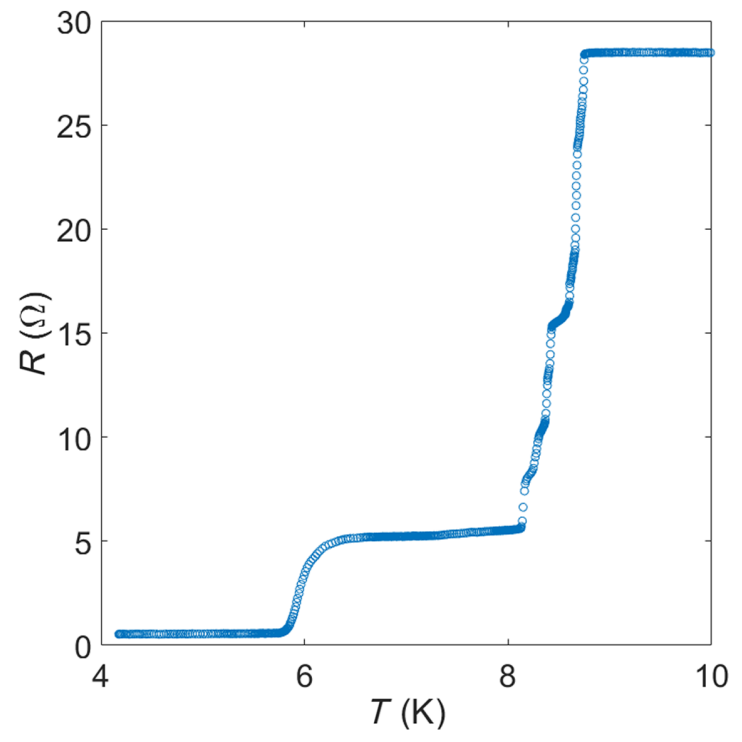

FIG. 5. Resistance versus temperature measurement showing $T_{c}$ of the SQUID-on-lever and the residual resistance of the bonding wires and contacts below $5.8 \mathrm{~K}$.

device. Figure 5 shows a resistance versus temperature measurement, which is made while cooling the SQUIDon-lever down with a constant applied bias current of $1 \mu \mathrm{A}$.

The as-deposited $\mathrm{Nb}$ film in the leads of the device becomes superconducting around $8.8 \mathrm{~K}$, as seen in the higher-temperature transition of Fig. 5. The lowertemperature transition at $5.8 \mathrm{~K}$ corresponds to the FIBpatterned parts of the SQUID-on-lever probe becoming superconducting. These regions of the $\mathrm{Nb}$ film are contaminated with implanted $\mathrm{Ga}$ ions, resulting in a decrease in $T_{c}$. The resistance of the device below $5.8 \mathrm{~K}$ is $0.5 \Omega$, which corresponds to the resistance of the bonding wires and contacts.

\section{Magnetic field dependence and magnetic sensitivity}

Figure 6 shows the differential resistance of the SQUID $R_{d}=d V_{\mathrm{SQUID}} / d I_{\mathrm{SQUID}}$ as a function of $B_{a}$ and $I_{\mathrm{SQUID}}$ obtained from the measured $I_{\text {SQUID }}$ vs $V_{b}$ curves. The SQUID shows quantum interference up to $B_{a}=1 \mathrm{~T}$. The interference pattern has a period $\Delta B=20 \mathrm{mT}$, which corresponds to an effective SQUID loop diameter $d_{e}=$ $2 \sqrt{\Phi_{0} / \pi \Delta B}=365 \mathrm{~nm}$, where $\Phi_{0}$ is the magnetic flux quantum.

The magnetic sensitivity of the device is obtained from the magnetic response function $d I_{\text {SQUID }} / d B$ and the white noise floor of the device. The magnetic response is calculated from $I_{\mathrm{SQUID}}\left(V_{b}\right)$ measured at different applied magnetic fields $B_{a}$. Figure 7(a) shows a plot of magnetic response with applied magnetic field at a fixed bias $V_{b}=$ $0.4 \mathrm{~V}$. At this constant bias voltage, the spectral density of the current noise $S_{I}^{1 / 2}$ at $10 \mathrm{kHz}$ is measured for the same 


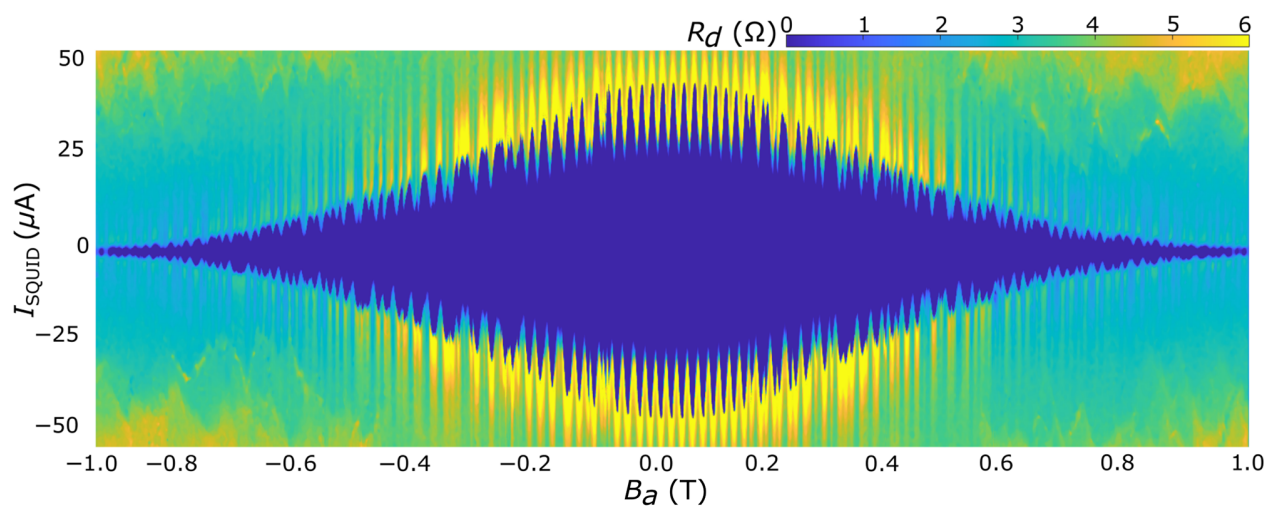

FIG. 6. Differential resistance $R_{d}=d V_{\text {SQUID }} / d I_{\text {SQUID }}$ as a function of $B_{a}$ and $I_{\mathrm{SQUID}}$ of the device showing SQUID interference pattern up to $1 \mathrm{~T}$. range of $B_{a}$, as shown in Fig. 7(b). The magnetic field noise of the device is then given by $S_{B}^{1 / 2}=S_{I}^{1 / 2} /\left|d I_{\mathrm{SQUID}} / d B\right|$. The corresponding flux noise is obtained from $S_{\Phi}^{1 / 2}=$ $S_{B}^{1 / 2} \times\left(A / \Phi_{0}\right)$, where $\Phi_{0}$ is the flux quantum and $A$ is the effective area of the SQUID loop. The field and flux noises of the device are shown as a function of $B_{a}$ in Figs. 7 (c) and 7(d), respectively. Both decrease in the regions of the interference pattern with a strong magnetic response. In the most sensitive regions, the device attains a field sensitivity $S_{B}^{1 / 2}=9.5 \mathrm{nT} / \sqrt{\mathrm{Hz}}$ or flux sensitivity $S_{\Phi}^{1 / 2}=$ $0.48 \mu \Phi_{0} / \sqrt{\mathrm{Hz}}$.

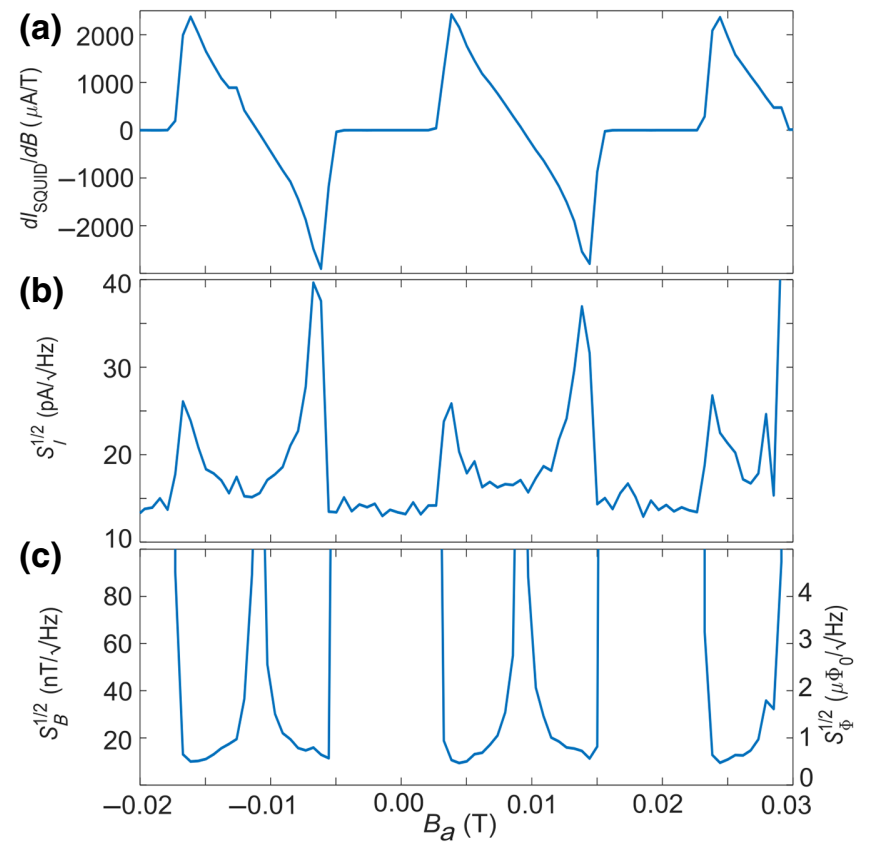

FIG. 7. (a) Magnetic response function $d I_{\text {SQUID }} / d B$ and (b) current noise $S_{I}^{1 / 2}$ at a constant bias voltage $V_{b}=0.4 \mathrm{~V}$ plotted as a function of applied magnetic field $B_{a}$. (c) Corresponding field noise and flux noise of the SQUID-on-lever probe as a function of $B_{a}$.

\section{Noise limits}

Thermal or Johnson noise of a SQUID is given by $S_{\Phi}^{1 / 2}=\sqrt{4 k_{B} T L(\pi L C)^{1 / 2}}$, where $k_{B}$ is the Boltzmann constant, $T$ is the temperature, $L$ is the inductance, and $C$ is the capacitance of the SQUID. The Dayem bridge JJs used here have small capacitances, such that the capacitance of the SQUID-on-lever probe is dominated by the capacitance of the two FIB-cut electrical leads running from the base of the cantilever to its apex. We estimate the total capacitance of the device by measuring the hysteresis in $I_{c}$ of the device as a function of $V_{b}$ [44]. By ignoring the effects of possible self-heating in the JJs, this procedure gives us an upper bound on $C=0.65 \mathrm{pF}$. We estimate the screening parameter $\beta_{L}$ from the modulation of $I_{c}$ assuming symmetric JJs. From $\beta_{L}=I_{C} L / \Phi_{0}$, we calculate the device's inductance as $L=32 \mathrm{pH}$ [44]. From these estimates, we calculate an approximate upper limit for the thermal noise $S_{\Phi}^{1 / 2}=120 \mathrm{n} \Phi_{0} / \sqrt{\mathrm{Hz}}$ at $4.2 \mathrm{~K}$. Given that the quantum noise $S_{\Phi}^{1 / 2}=\sqrt{\hbar L}$, where $\hbar$ is the Planck constant, we estimate $S_{\Phi}^{1 / 2}=27 \mathrm{n} \Phi_{0} / \sqrt{\mathrm{Hz}}$.

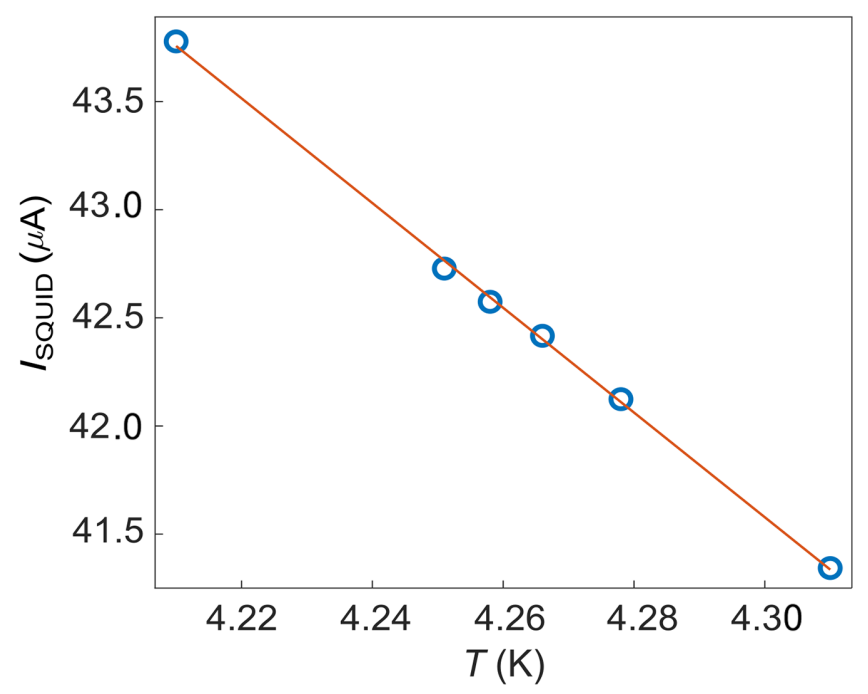

FIG. 8. $I_{\text {SQUID }}$ versus temperature $T$ at $V_{b}=0.55 \mathrm{~V}$. Thermal response of $-24.21 \mu \mathrm{A} / \mathrm{K}$ is obtained from the slope. 
The measured noise floor of $S_{\Phi}^{1 / 2}=0.48 \mu \Phi_{0} / \sqrt{\mathrm{Hz}}$ exceeds both of these estimates, meaning that our measurement is likely limited by nonfundamental noise such as interference or electrical noise. We therefore expect that the noise can be improved until it reaches these fundamental limits.

\section{Thermal sensitivity}

In order to determine the thermal sensitivity of the device, we fix the voltage bias to make the SQUID-onlever probe maximally sensitive to changes in temperature. As shown in Fig. 2(d) of the main text, where $I_{\text {SQUID }}\left(V_{b}\right)$ is measured at $B_{a}=0$ and different $T$, this occurs for $V_{b}=0.55 \mathrm{~V}$. At this bias voltage, we plot $I_{\mathrm{SQUID}}(T)$ in Fig. 8. The slope of this curve yields the thermal response of the device $d I_{\mathrm{SQUID}} / d T=-24.2 \mu \mathrm{A} / \mathrm{K}$ at $4.2 \mathrm{~K}$. By measuring the white current noise $S_{I}^{1 / 2}$, we determine the temperature noise $S_{T}^{1 / 2}=S_{I}^{1 / 2} /\left|d I_{\text {SQUID }} / d T\right|$ of the device. The SQUID-on-lever probe reaches a thermal sensitivity $S_{T}^{1 / 2}=620 \mathrm{nK} / \sqrt{\mathrm{Hz}}$ at zero magnetic field and $4.2 \mathrm{~K}$.

\section{APPENDIX C: TIP-SAMPLE DISTANCE CALIBRATION}

Figure 9(a) shows an approach curve, i.e., a measurement of the cantilever frequency shift $\Delta f$ as a function of the tip-sample spacing $z$. The sample being approached is the current-carrying wire imaged in Fig. 3. $z=0$ is determined by the spacing at which the cantilever tip comes (a)

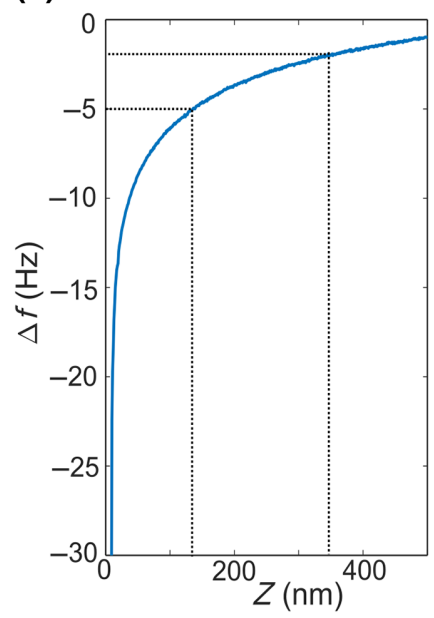

(b) $B_{z}^{\mathrm{ac}}(\mu \mathrm{T})-20-10 \quad 0 \quad 10 \quad 20$

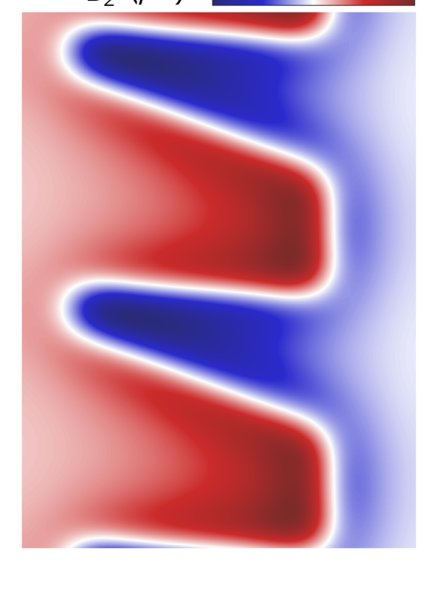

FIG. 9. (a) Approach curve showing frequency shift $\Delta f$ as a function of tip-sample spacing $z$. Dotted lines indicate the scanning height for images of the current-carrying wire shown in Fig. 3(b) for $\Delta f=-2 \mathrm{~Hz}$ and in Figs. 3(c)-3(e) for $\Delta f=-5$ Hz. (b) Simulation of the Biot-Savart field produced by the current-carrying wire at a SQUID-sample height $z_{\text {SQUID }}=520$ $\mathrm{nm}$. into contact with the surface. At this spacing, the signal from the interferometer, which measures the cantilever displacement, shows a sudden discrete change.

The noncontact AFM image shown in Fig. 3(b) is obtained at a set-point $\Delta f=-5 \mathrm{~Hz}$, which-according to the approach curve in Fig. 9(a)-corresponds to a tipsample spacing $z=140 \mathrm{~nm}$ and a SQUID-sample spacing $z_{\text {SQUID }}=315 \mathrm{~nm}$ (the height of the protruding tip is $175 \mathrm{~nm}$ ). The images of $B_{z}$ shown in Figs. 3(c)-3(e) are obtained at a set-point of $\Delta f=-2 \mathrm{~Hz}$, which corresponds to $z=345 \mathrm{~nm}$ and $z_{\text {SQUID }}=520 \mathrm{~nm}$. Both distances are calibrated via the approach curve.

We check this calibration by simulating the Biot-Savart field produced by the current-carrying wire at $z_{\text {SQUID }}=$ $520 \mathrm{~nm}$ and comparing it with the measurement of $B_{z}^{\mathrm{ac}}$ in Fig. 3(c). We use COMSOL Multiphysics $5.5 \mathrm{ac} / \mathrm{dc}$ module to simulate the current density distribution inside the gold wire originating from an applied current of $100 \mu \mathrm{A}$. The simulated geometry of the wire matches that of the measured sample and we apply typical parameters for $\mathrm{Au}$ at $T=4.2 \mathrm{~K}$. We then calculate the Biot-Savart field produced by the current density and extract the $z$ component of the magnetic field $B_{z}^{\text {ac }}$. For these simulations we use a custom tetrahedral mesh with a minimum element size of $80 \mathrm{~nm}$. We convolve the simulated magnetic field with a Gaussian point-spread function with standard deviation $\sigma=365 \mathrm{~nm}$, in order to account for the size of the SQUID loop. In Fig. 9(b), we show the simulation of $B_{z}^{\text {ac }}$ at the SQUID-sample spacing of the measurement. This simulation matches the measurement shown in Fig. 3(c) of the main text, confirming the scanning height obtained from the approach curve.

\section{APPENDIX D: SPATIAL RESOLUTION}

We estimate the spatial resolution of our sensor in magnetic imaging mode by comparing $B_{z}(x, y)$ profiles measured using the SQUID-on-lever probe above the artificial spin ice sample with simulations of these field profiles convolved with a Gaussian point-spread function with different standard deviations $\sigma$. Figure 10(a) shows the measured $B_{z}(x, y)$ with the SQUID $z_{\text {SQUID }}=450 \mathrm{~nm}$ above the substrate. Figure 10(b) shows the corresponding simulation of $B_{z}(x, y)$ without any Gaussian smoothing, i.e., $\sigma=0$. Corresponding horizontal and vertical line cuts through the measured and simulated $B_{z}(x, y)$ are shown in Figs. 10(c) and 10(d), respectively. The measured line cuts are plotted as open squares, while colored lines show simulated line cuts with various degrees of Gaussian smoothing, indicated in the legend. For the horizontal and vertical line cuts, the Gaussian smoothing corresponding to the minimum mean squared deviation from the measured data is plotted as a yellow line. This smoothing corresponds to a standard deviation of $\sigma_{x}=180 \pm 10 \mathrm{~nm}$ in the horizontal direction and $\sigma_{y}=180 \pm 20 \mathrm{~nm}$ in the vertical 
(a)

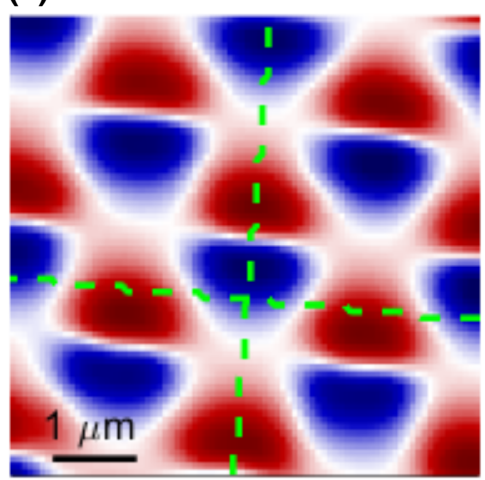

(b)

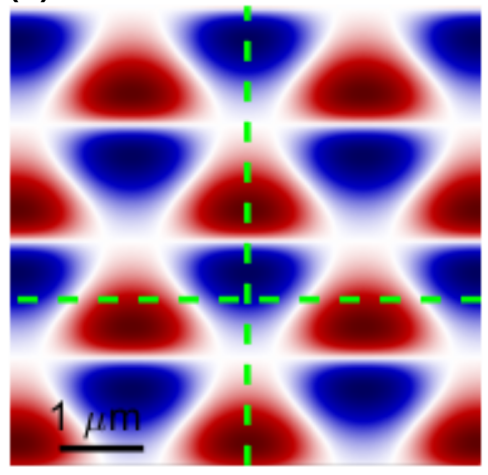

(c)

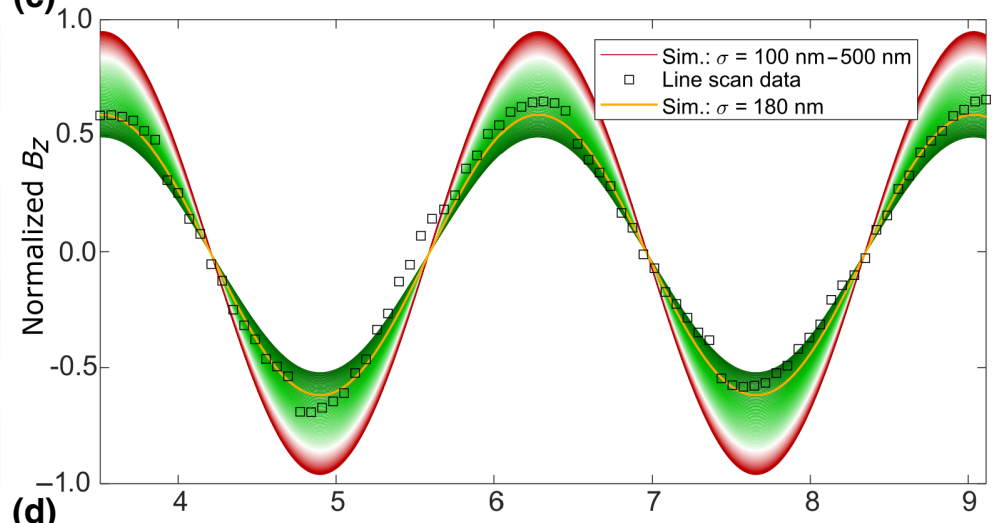

(d)

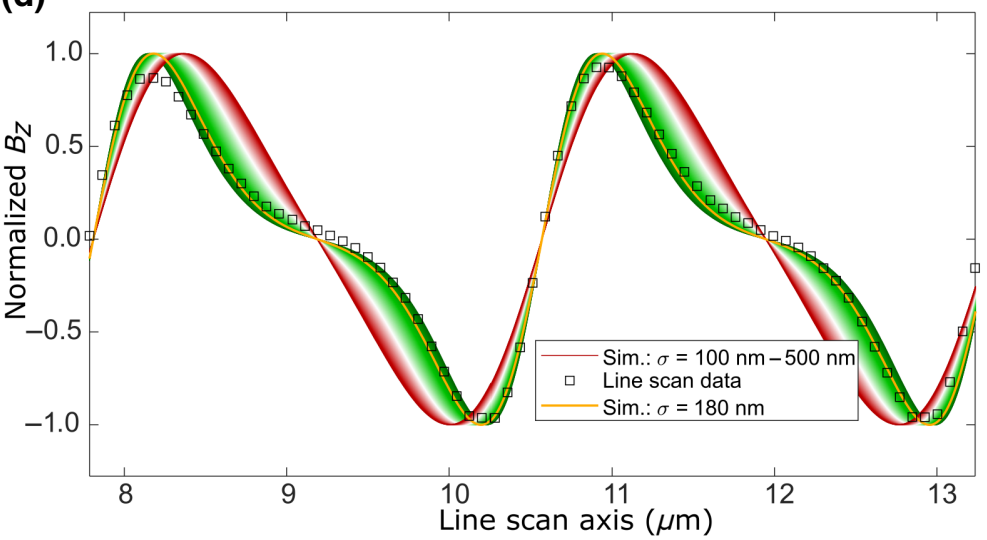

FIG. 10. (a) A region of $B_{z}(x, y)$ measured with the SQUID $z_{\text {SQUID }}=450 \mathrm{~nm}$ above the substrate of the artificial spin ice sample at remanence. (b) Corresponding simulation of $B_{z}(x, y)$ without Gaussian smoothing $(\sigma=0)$. Measured $B_{z}$ shown as open squares along the horizontal (c) and vertical (d) line cuts corresponding to the green dashed lines in (a),(b). Simulated $B_{z}$ is shown along the same line cuts for different degrees of Gaussian smoothing, as indicated in the legend. Plots in (c),(d) are normalized to the maximum $B_{z}$.

direction. The error represents the range within which the mean squared deviation remains within $10 \%$ of the minimum. The resulting two-dimensional Gaussian pointspread function is symmetric within the error. If we take a two-dimensional Gaussian with the larger of the two standard deviations, the spatial resolution of our probe can be described by a Gaussian point-spread function with a $\mathrm{FWHM} \simeq 2.355 \sigma=420 \pm 30 \mathrm{~nm}$. This magnetic spatial resolution is close to but larger than $d_{e}=365 \mathrm{~nm}$ of the SQUID and smaller than the 900-nm width of the triangular superconducting plateau.

This magnetic imaging measurement is carried out with a SQUID-sample spacing $z_{\text {SQUID }}=450 \mathrm{~nm}$. In this case, as in other magnetic images shown in the paper, $z_{\text {SQUID }} \gtrsim$ $d_{e}$, such that a symmetric Gaussian point-spread function appropriately describes the imaging process. Upon close approach, where $z_{\mathrm{SQUID}}<d_{e}$, such a simple point-spread function is no longer adequate. A more complex pointspread function is necessary that takes into account the details of the flux focusing effect of the roughly triangular superconducting film around the SQUID loop, visible in Fig. 1(c). In this close-approach limit, magnetic images derived from the scanning SQUID-on-lever probe are better plotted in terms of magnetic flux threading through the device, rather than magnetic field, given that the sensor's point-spread function alters the image in a more complex way than simple Gaussian smoothing [17].

[1] E. Marchiori, L. Ceccarelli, N. Rossi, L. Lorenzelli, C. L. Degen, and M. Poggio, Nanoscale magnetic field imaging for 2D materials, Nat. Rev. Phys. 4, 49 (2022).

[2] K. H. Kuit, J. R. Kirtley, W. van der Veur, C. G. Molenaar, F. J. G. Roesthuis, A. G. P. Troeman, J. R. Clem, H. Hilgenkamp, H. Rogalla, and J. Flokstra, Vortex trapping and expulsion in thin-film $\mathrm{YBa}_{2} \mathrm{Cu}_{3} \mathrm{O}_{7}-\delta$ strips, Phys. Rev. B 77, 134504 (2008).

[3] L. Embon, Y. Anahory, A. Suhov, D. Halbertal, J. Cuppens, A. Yakovenko, A. Uri, Y. Myasoedov, M. L. Rappaport, M. E. Huber, A. Gurevich, and E. Zeldov, Probing dynamics and pinning of single vortices in superconductors at nanometer scales, Sci. Rep. 5, 7598 (2015).

[4] L. Ceccarelli, D. Vasyukov, M. Wyss, G. Romagnoli, N. Rossi, L. Moser, and M. Poggio, Imaging pinning 
and expulsion of individual superconducting vortices in amorphous MoSi thin films, Phys. Rev. B 100, 104504 (2019).

[5] I. P. Zhang, J. C. Palmstrom, H. Noad, L. Bishop-Van Horn, Y. Iguchi, Z. Cui, E. Mueller, J. R. Kirtley, I. R. Fisher, and K. A. Moler, Imaging anisotropic vortex dynamics in FeSe, Phys. Rev. B 100, 024514 (2019).

[6] H. Bluhm, N. C. Koshnick, J. A. Bert, M. E. Huber, and K. A. Moler, Persistent Currents in Normal Metal Rings, Phys. Rev. Lett. 102, 136802 (2009).

[7] Julie A. Bert, Beena Kalisky, Christopher Bell, Minu Kim, Yasuyuki Hikita, Harold Y. Hwang, and Kathryn A. Moler, Direct imaging of the coexistence of ferromagnetism and superconductivity at the $\mathrm{LaAlO}_{3} / \mathrm{SrTiO}_{3}$ interface, Nat. Phys. 7, 767 (2011).

[8] Y. Anahory, L. Embon, C. J. Li, S. Banerjee, A. Meltzer, H. R. Naren, A. Yakovenko, J. Cuppens, Y. Myasoedov, M. L. Rappaport, M. E. Huber, K. Michaeli, T. Venkatesan, Ariando, and E. Zeldov, Emergent nanoscale superparamagnetism at oxide interfaces, Nat. Commun. 7, 12566 (2016).

[9] D. Vasyukov, L. Ceccarelli, M. Wyss, B. Gross, A. Schwarb, A. Mehlin, N. Rossi, G. Tütüncüoglu, F. Heimbach, R. R. Zamani, A. Kovács, A. Fontcuberta i Morral, D. Grundler, and M. Poggio, Imaging stray magnetic field of individual ferromagnetic nanotubes, Nano Lett. 18, 964 (2018).

[10] M. Wyss, S. Gliga, D. Vasyukov, L. Ceccarelli, G. Romagnoli, J. Cui, A. Kleibert, R. L. Stamps, and M. Poggio, Stray-field imaging of a chiral artificial spin ice during magnetization reversal, ACS Nano 13, 13910 (2019).

[11] A. Uri, Y. Kim, K. Bagani, C. K. Lewandowski, S. Grover, N. Auerbach, E. O. Lachman, Y. Myasoedov, T. Taniguchi, K. Watanabe, J. Smet, and E. Zeldov, Nanoscale imaging of equilibrium quantum Hall edge currents and of the magnetic monopole response in graphene, Nat. Phys. 16, 164 (2020).

[12] K. C. Nowack, E. M. Spanton, M. Baenninger, M. König, J. R. Kirtley, B. Kalisky, C. Ames, P. Leubner, C. Brüne, H. Buhmann, L. W. Molenkamp, D. Goldhaber-Gordon, and K. A. Moler, Imaging currents in HgTe quantum wells in the quantum spin Hall regime, Nat. Mater. 12, 787 (2013).

[13] A. Uri, S. Grover, Y. Cao, J. A. Crosse, K. Bagani, D. Rodan-Legrain, Y. Myasoedov, K. Watanabe, T. Taniguchi, P. Moon, M. Koshino, P. Jarillo-Herrero, and E. Zeldov, Mapping the twist-angle disorder and landau levels in magic-angle graphene, Nature 581, 47 (2020).

[14] C. L. Tschirhart, M. Serlin, H. Polshyn, A. Shragai, Z. Xia, J. Zhu, Y. Zhang, K. Watanabe, T. Taniguchi, M. E. Huber, and A. F. Young, Imaging orbital ferromagnetism in a moiréChern insulator, Science 372, 1323 (2021).

[15] J. R. Kirtley, M. B. Ketchen, K. G. Stawiasz, J. Z. Sun, W. J. Gallagher, S. H. Blanton, and S. J. Wind, High-resolution scanning SQUID microscope, Appl. Phys. Lett. 66, 1138 (1995).

[16] N. C. Koshnick, M. E. Huber, J. A. Bert, C. W. Hicks, J. Large, H. Edwards, and K. A. Moler, A terraced scanning super conducting quantum interference device susceptometer with submicron pickup loops, Appl. Phys. Lett. 93, 243101 (2008).

[17] J. R. Kirtley, L. Paulius, A. J. Rosenberg, J. C. Palmstrom, C. M. Holland, E. M. Spanton, D. Schiessl, C. L. Jermain, J. Gibbons, Y.-K.-K. Fung, M. E. Huber, D. C. Ralph, M. B. Ketchen, G. W. Gibson, and K. A. Moler, Scanning SQUID susceptometers with sub-micron spatial resolution, Rev. Sci. Instrum. 87, 093702 (2016).

[18] A. Finkler, Y. Segev, Y. Myasoedov, M. L. Rappaport, L. Ne'eman, D. Vasyukov, E. Zeldov, M. E. Huber, J. Martin, and A. Yacoby, Self-aligned nanoscale SQUID on a tip, Nano Lett. 10, 1046 (2010).

[19] A. Finkler, D. Vasyukov, Y. Segev, L. Ne'eman, E. O. Lachman, M. L. Rappaport, Y. Myasoedov, E. Zeldov, and M. E. Huber, Scanning superconducting quantum interference device on a tip for magnetic imaging of nanoscale phenomena, Rev. Sci. Instrum. 83, 073702 (2012).

[20] D. Vasyukov, Y. Anahory, L. Embon, D. Halbertal, J. Cuppens, L. Neeman, A. Finkler, Y. Segev, Y. Myasoedov, M. L. Rappaport, M. E. Huber, and E. Zeldov, A scanning superconducting quantum interference device with single electron spin sensitivity, Nat. Nanotechnol. 8, 639 (2013).

[21] D. Halbertal, J. Cuppens, M. Ben Shalom, L. Embon, N. Shadmi, Y. Anahory, H. R. Naren, J. Sarkar, A. Uri, Y. Ronen, Y. Myasoedov, L. S. Levitov, E. Joselevich, A. K. Geim, and E. Zeldov, Nanoscale thermal imaging of dissipation in quantum systems, Nature 539, 407 (2016).

[22] D. Halbertal, M. Ben Shalom, A. Uri, K. Bagani, A. Y. Meltzer, I. Marcus, Y. Myasoedov, J. Birkbeck, L. S. Levitov, A.e K. Geim, and E. Zeldov, Imaging resonant dissipation from individual atomic defects in graphene, Science 358, 1303 (2017).

[23] B. W. Gardner, J. C. Wynn, P. G. Björnsson, E. W. J. Straver, K. A. Moler, J. R. Kirtley, and M. B. Ketchen, Scanning superconducting quantum interference device susceptometry, Rev. Sci. Instrum. 72, 2361 (2001).

[24] M. E. Huber, N. C. Koshnick, H. Bluhm, L. J. Archuleta, T. Azua, P. G. Björnsson, B. W. Gardner, S. T. Halloran, E. A. Lucero, and K. A. Moler, Gradiometric micro-SQUID susceptometer for scanning measurements of mesoscopic samples, Rev. Sci. Instrum. 79, 053704 (2008).

[25] See Appendix B.

[26] K. Bagani, J. Sarkar, A. Uri, M. L. Rappaport, M. E. Huber, E. Zeldov, and Y. Myasoedov, Sputtered $\mathrm{Mo}_{6} 6 \mathrm{Re}_{3} 4$ SQUID-On-Tip for High-Field Magnetic and Thermal Nanoimaging, Phys. Rev. Appl. 12, 044062 (2019).

[27] B. J. Roth, N. G. Sepulveda, and J. P. Wikswo, Using a magnetometer to image a two-dimensional current distribution, J. Appl. Phys. 65, 361 (1989).

[28] K. Chang, A. Eichler, J. Rhensius, L. Lorenzelli, and C. L. Degen, Nanoscale imaging of current density with a singlespin magnetometer, Nano Lett. 17, 2367 (2017).

[29] R. F. Wang, C. Nisoli, R. S. Freitas, J. Li, W. McConville, B. J. Cooley, M. S. Lund, N. Samarth, C. Leighton, V. H. Crespi, and P. Schiffer, Artificial spin ice in a geometrically frustrated lattice of nanoscale ferromagnetic islands, Nature 439, 303 (2006). 
[30] S. H. Skjærvø, C. H. Marrows, R. L. Stamps, and L. J. Heyderman, Advances in artificial spin ice, Nat. Rev. Phys. 2, 13 (2020).

[31] C. Phatak, A. K. Petford-Long, O. Heinonen, M. Tanase, and M. De Graef, Nanoscale structure of the magnetic induction at monopole defects in artificial spin-ice lattices, Phys. Rev. B 83, 174431 (2011).

[32] E. Y. Vedmedenko, Dynamics of Bound Monopoles in Artificial Spin Ice: How to Store Energy in Dirac Strings, Phys. Rev. Lett. 116, 077202 (2016).

[33] A. Farhan, P. M. Derlet, A. Kleibert, A. Balan, R. V. Chopdekar, M. Wyss, J. Perron, A. Scholl, F. Nolting, and L. J. Heyderman, Direct Observation of Thermal Relaxation in Artificial Spin Ice, Phys. Rev. Lett. 111, 057204 (2013).

[34] A. Farhan, M. Saccone, C. F. Petersen, S. Dhuey, R. V. Chopdekar, Y.-L. Huang, N. Kent, Z. Chen, M. J. Alava, T. Lippert, A. Scholl, and S. van Dijken, Emergent magnetic monopole dynamics in macroscopically degenerate artificial spin ice, Sci. Adv. 5, 6380 (2019).

[35] S. Gliga, E. Iacocca, and O. G. Heinonen, Dynamics of reconfigurable artificial spin ice: Toward magnonic functional materials, APL Mater. 8, 040911 (2020).

[36] M. T. Kaffash, S. Lendinez, and M. B. Jungfleisch, Nanomagnonics with artificial spin ice, Phys. Lett. A 402, 127364 (2021).

[37] T. Dion, J. C. Gartside, A. Vanstone, K. D. Stenning, D. M. Arroo, H. Kurebayashi, and W. R. Branford, Observation and control of collective spin-wave mode hybridization in chevron arrays and in square, staircase, and brickwork artificial spin ices, Phys. Rev. Research 4, 013107 (2022).

[38] Y. Shibata, S. Nomura, H. Kashiwaya, S. Kashiwaya, R. Ishiguro, and H. Takayanagi, Imaging of current density distributions with a $\mathrm{Nb}$ weak-link scanning nano-SQUID microscope, Sci. Rep. 5, 15097 (2015).

[39] Y. Frenkel, N. Haham, Y. Shperber, C. Bell, Y. Xie, Z. Chen, Y. Hikita, H. Y. Hwang, E. K. H. Salje, and B. Kalisky, Imaging and tuning polarity at $\mathrm{SrTiO}_{3}$ domain walls, Nat. Mater. 16, 1203 (2017).

[40] R. Córdoba, T. I. Baturina, J. Sesé, A. Yu Mironov, J. M. De Teresa, M. R. Ibarra, D. A. Nasimov, A. K. Gutakovskii, A. V. Latyshev, I. Guillamón, H. Suderow, S. Vieira, M. R. Baklanov, J. J. Palacios, and V. M. Vinokur, Magnetic field-induced dissipation-free state in superconducting nanostructures, Nat. Commun. 4, 1437 (2013).

[41] B. Müller, M. Karrer, F. Limberger, M. Becker, B. Schröppel, C. J. Burkhardt, R. Kleiner, E. Goldobin, and D. Koelle, Josephson Junctions and SQUIDs Created by Focused Helium-Ion-Beam Irradiation of $\mathrm{YBa}_{2} \mathrm{Cu}_{3} \mathrm{O}_{7}$, Phys. Rev. Appl. 11, 044082 (2019).

[42] http://scicore.unibas.ch/.

[43] A. Vansteenkiste, J. Leliaert, M. Dvornik, M. Helsen, F. Garcia-Sanchez, and B. Van Waeyenberge, The design and verification of MuMax3, AIP Adv. 4, 107133 (2014).

[44] The SQUID Handbook Vol. 1: Fundamentals and Technology of SQUIDs and SQUID Sytems, J. Clarke, A. Braginski, Eds., Wiley (2004). 